Annuaire suisse de politique de développement

$19 \mid 2000$

Pour une politique plus cohérente envers les pays en développement

\title{
La cohérence des politiques : une nouvelle approche des relations Nord-Sud
}

Jacques Forster

\section{OpenEdition}

\section{Journals}

Édition électronique

URL : http://journals.openedition.org/aspd/833

DOI : $10.4000 /$ aspd. 833

ISSN : 1663-9669

Éditeur

Institut de hautes études internationales et du développement

Édition imprimée

Date de publication : 1 avril 2000

Pagination : $3-11$

ISSN : 1660-5934

Référence électronique

Jacques Forster, «La cohérence des politiques : une nouvelle approche des relations Nord-Sud », Annuaire suisse de politique de développement [En ligne], 19 | 2000, mis en ligne le 15 août 2012.

consulté le 08 septembre 2020. URL : http://journals.openedition.org/aspd/833 ; DOI : https://doi.org/ $10.4000 /$ aspd. 833 


\title{
LA COHÉRENCE DES POLITIQUES: UNE NOUVELLE APPROCHE DES RELATIONS NORD-SUD
}

\author{
JACQUES FORSTER*
}

\section{INTRODUCTION}

En 1991, le Comité d'aide au développement (CAD) de l'OCDE inscrivait à l'ordre du jour de sa réunion ministérielle le thème de la cohérence des politiques des pays membres vis-à-vis des pays en développement. Il proposait ainsi une nouvelle approche des relations Nord-Sud qui prenne en compte les effets sur le développement des divers volets de ces relations et, tout particulièrement, les effets des politiques des pays industrialisés. Cette démarche donnait une nouvelle actualité à un souci exprimé depuis de nombreuses années par les milieux non gouvernementaux. Elle remettait aussi en mémoire la formule des années 1960: trade, not aid, référence lapidaire au rôle des politiques commerciales dans la promotion du développement des pays du Tiers Monde.

\section{Nous examinons successivement dans cet article ${ }^{1}$}

- quel contenu est aujourd'hui donné à ce concept de «cohérence des politiques»;

- la genèse de cette approche;

- les obstacles auxquels elle se heurte;

- les mécanismes de sa mise œuvre;

- son potentiel et ses limites.

\section{QU'EST-CE QUE LA COHÉRENCE DES POLITIQUES?}

Bien que le concept de «cohérence des politiques» n'ait pas - ou pas encore - sa place dans la science politique, la problématique qu'il sous-tend a toujours été présente dans la réflexion sur la coopération internationale au développement ${ }^{2}$. Ce thème a en effet été débattu dans les milieux universitaires et des ONG dès les années 1960; les gouvernements étaient alors assez réticents à les suivre pour des raisons qui seront évoquées dans la troisième partie de cet article $^{3}$.

* Professeur à l'IUED.

1. Cet article est issu d'une recherche collective menée en 1997 et 1998 avec l'appui de la DDC. Le résultat complet de cette recherche est publié dans un ouvrage collectif issu d'un colloque qui a eu lieu à Genève en avril 1997. Voir J. Forster and O. Stokke (eds), Policy Coherence in Development Co-operation, EADI Book Series number 22, Frank Cass, London 1999, 499 p.

2. G. Hyden, «The Shifting Ground of Policy Coherence in Development Co-operation », in J. Forster and O. Stokke, op. cit., pp. 58 et 59 .

3. En Suisse, dès 1975, des ONG publiaient un rapport, Maldéveloppement Suisse-Monde, selon lequel l'incompatibilité entre la politique économique extérieure et la politique de coopération au développement devait être discutée ouvertement. 
Fukasaku et Hirata vont directement au cœur de la problématique en définissant l'approche de la cohérence comme l'examen des politiques et de leurs instruments sous l'angle de leurs effets combinés sur les pays en développement ${ }^{4}$. Cette définition du concept de cohérence ne suffit cependant pas à le rendre opératoire. Encore faut-il s'entendre sur des paramètres tels que

1) la finalité de la cohérence des politiques (cohérence pour quoi et pour qui ?);

2) la cohérence entre la formulation et la mise en œuvre des politiques;

3) les niveaux auxquels la cohérence est recherchée:

- au sein d'une seule politique (par exemple de coopération au développement);

- entre plusieurs politiques (d'aide, commerciale, migratoire, relative à l'environnement, etc.).

1) La finalité de la cohérence des politiques vis-à-vis des pays en développement est, selon notre perspective, de veiller à ce que les politiques des pays industrialisés favorisent le développement économique et social des pays en développement ou tout au moins ne lui nuisent pas. Cela soulève la question du type de développement que les politiques Nord-Sud des pays industrialisés entendent promouvoir. A première vue, il semble régner depuis une dizaine d'années un large consensus sur les objectifs de la coopération. Celle-ci cherche généralement à encourager

- la croissance économique reposant sur une économie ouverte;

- l'équité sociale;

$\checkmark$ une gestion durable des ressources naturelles;

- la démocratie et les droits de l'homme;

- la prévention des conflits et la promotion de la paix.

Ce consensus n'exclut cependant pas des divergences quant à la priorité à accorder à chacun de ces objectifs ou quant à la façon de résoudre les conflits entre certains objectifs. Les exemples de tels conflits sont nombreux: les tensions sont fréquentes entre l'objectif de croissance et celui d'équité ou celui qui veut promouvoir une gestion durable des ressources naturelles.

De même, la relation entre croissance économique et droits de l'homme est souvent à l'ordre du jour des débats sur la cohérence, car les pays industriels sont tous confrontés à la question suivante: la coopération économique internationale doit-elle promouvoir la croissance économique sans prendre en considération la situation des droits de l'homme, en adoptant l'hypothèse que le progrès économique favorisera un plus grand respect des droits de l'homme? Selon la réponse apportée à cette question, les mêmes politiques économiques extérieures et des droits de l'homme seront jugées cohérentes ou incohérentes les unes par rapport aux autres. Il n'y a pas de réponse simple à ces dilemmes politiques, et cette question montre combien il est nécessaire pour les gouvernements d'être très explicites non seulement sur les objectifs de leur politique de développement mais aussi sur les relations entre ces objectifs.

4. K. Fukasaku and A. Hirata, The OECD and the ASEAN Economies: The Challenge of Policy Coherence, OECD Development Centre, Paris, 1995, p. 20. 
2) Formulation et mise en $2 u v r e$ des politiques. Dans le contexte des relations Nord-Sud, ce sont bien les contradictions et les complémentarités entre les objectifs de différentes politiques qui attirent le plus l'attention. Une autre dimension est cependant importante, celle de la cohérence entre la formulation des politiques et leur mise en œuvre. Des lacunes dans la mise en œuvre d'une politique peuvent être attribuées à un manque de volonté ou à une insuffisance de moyens; dans les deux cas, la perception est celle d'un échec qui peut affecter la légitimité des pouvoirs publics. L'analyse de la cohérence des politiques doit donc aussi s'intéresser à la mise en œuvre de ces politiques. On pourrait cependant penser que, dans le cas de la politique de développement, la question de la légitimité est relativement peu importante car les principaux stakeholders ne font pas partie de la scène politique nationale. Le coût politique de l'incohérence serait ainsi moindre dans ce domaine que dans d'autres ${ }^{5}$.

3) Les types de cohérence. La cohérence des politiques peut être envisagée à différents niveaux et l'analyse peut se situer à l'échelle nationale ou internationale.

A l'échelle d'un pays industrialisé, on peut analyser la cohérence à deux niveaux :

- au niveau de la politique de coopération au développement elle-même; il s'agit donc de la cohérence interne de cette politique. Des contradictions peuvent apparaître entre les objectifs et les moyens de cette politique (par exemple la politique de participation des partenaires locaux aux décisions et les moyens mis en œuvre pour assurer cette participation), ou entre différents objectifs (par exemple ceux de l'ajustement structurel et du développement social);

- au niveau de la cohérence entre la politique de coopération au développement et d'autres politiques. On peut faire une distinction entre, d'une part, les politiques qui concernent directement les pays en développement (commerce, investissement international, migrations, droits de l'homme, etc.), et, d'autre part, les politiques des domaines tels que l'environnement, l'agriculture, la défense, qui ne touchent pas directement les pays du Sud mais peuvent néanmoins avoir des effets sur leur développement. A ce niveau, c'est aussi la crédibilité des pays industriels qui est en jeu. En effet, il peut y avoir des différences importantes entre les recommandations qu'ils font à leurs partenaires du Sud et leurs propres politiques; le protectionnisme agricole est un exemple de contradiction entre le discours et la pratique de certains pays industriels.

A l'échelle internationale, l'analyse de la cohérence peut concerner soit les politiques des divers pays industriels envers les pays en développement (il s'agit là du thème ancien et récurrent de la coordination des politiques des pays fournisseurs d'aide, élargi à d'autres domaines des relations Nord-Sud), soit l'harmonisation des politiques des pays en développement et des pays industriels pour que tous les efforts tendent vers des objectifs communs. Ce thème a pris une

5. Une illustration de cette problématique est fournie par la façon dont sont tranchées les situations où une politique cohérente vis-à-vis du Sud représenterait un coût - en termes d'emplois par exemple - dans le pays industriel. En Suisse, de tels dilemmes se présentent notamment dans des décisions concernant l'octroi de la GRE à des exportations pour des projets discutables sur le plan social ou environnemental. 
importance considérable ces dernières années sous l'appellation de policy dialogue - bilatéral ou multilatéral -, portant non seulement sur la politique économique, mais aussi sur les dimensions sociales et politiques du développement.

Dans le cadre de cette introduction à un examen de la cohérence des politiques Nord-Sud de la Suisse, nous aborderons ce thème uniquement au niveau national.

On peut enfin rappeler la distinction que fait Paul Hoebink ${ }^{6}$ entre l'incohérence consciente et l'incohérence non voulue. La première survient lorsque les décideurs publics acceptent consciemment que les objectifs d'une politique ne peuvent être atteints en raison de conflits d'intérêts. La seconde se produit lorsque des décisions «incohérentes » sont prises simplement par manque de communication entre différentes instances décisionnelles, et elle peut être corrigée par des mécanismes de coordination.

\section{L'ÉMERGENCE DE LA COHÉRENCE DES POLITIQUES}

Dans son acception actuelle, le thème de la cohérence des politiques émergea dans le débat international lors de la $30^{\mathrm{e}}$ réunion à haut niveau du $\mathrm{CAD}$ en décembre 1991. Une des conclusions de cette réunion fut que les objectifs de développement devaient être pris en compte dans toutes les dimensions des relations Nord-Sud (politiques macroéconomiques, commerciales, agricoles, ainsi que dans les domaines des migrations, de l'environnement, du commerce des armes, etc.). Il fut en outre recommandé que «l'administration dispose de mécanismes lui permettant de faire en sorte que les politiques ou décisions individuelles susceptibles d'entrer en conflit avec les objectifs à long terme [de développement] soient identifiées et soumises à un débat approprié» ${ }^{7}$. Quels facteurs favorisèrent cette nouvelle approche des relations Nord-Sud?

- La fin de la guerre froide a certainement été une condition préalable en permettant d'aborder les relations Nord-Sud dans une nouvelle perspective. Pour les protagonistes de la guerre froide, la promotion du développement au Sud était liée à des objectifs stratégiques, à la nécessité de soutenir des alliés ou de veiller à ce que les non-alignés ne basculent pas dans «l'autre camp ». La nouvelle donne internationale accorde plus de poids aux objectifs déclarés de la coopération au développement et permet aussi d'aborder dans le dialogue Nord-Sud des thèmes tels que les droits de l'homme, la bonne gestion des affaires publiques, les dépenses militaires. Auparavant, lorsque l'objectif était de maintenir des positions géostratégiques et de renforcer des alliés selon des critères qui n'étaient pas nécessairement ceux de leur contribution au développement de leur pays, ces thèmes étaient bannis des tables de négociations.

- La réduction des flux d'aide publique au développement qui a marqué les années 1990 est survenue à un moment où les tâches de coopération au déve-

6. P. Hoebink, «Coherence and Development Policy: The Case of the European Union », in J. Forster and O. Stokke, op. cit. p. 335.

7. OCDE, Coopération pour le développement 1992, OCDE, Paris, 1992, p. 55 
loppement s'élargissent et où la liste des pays bénéficiaires s'allonge (avec l'inclusion de pays postcommunistes dans la catégorie des pays en développement), créant une situation paradoxale pour les gouvernements du Nord confrontés au défi d'accroître l'efficacité des projets et programmes d'aide. L'approche de la cohérence des politiques est un instrument propre à assurer une meilleure gestion des ressources publiques en cherchant à éviter qu'une politique (commerciale par exemple) réduise ou annule les effets de la politique de coopération au développement ${ }^{8}$.

- La mondialisation de l'économie et l'intégration croissante des économies des pays en développement dans l'économie mondiale, suite aux programmes d'ajustement structurel et à l'Uruguay Round, ont nettement accru l'impact des politiques monétaires, financières et commerciales des pays industrialisés sur les pays du Sud. Il est donc plus important que jamais de connaître l'effet de ces politiques sur le développement économique et social de ces pays.

- La diversité et la complexité croissantes des relations Nord-Sud n'apparaissent pas seulement dans le domaine économique: les problèmes globaux liés à la détérioration de l'environnement, au développement de la criminalité internationale, à la production, distribution et consommation de narcotiques, à la disponibilité effrénée d'armes ont tous des dimensions Nord-Sud et sont traités par un grand nombre de ministères et d'agences gouvernementales. La politique de développement devient donc de plus en plus une tâche interministérielle, non seulement pour améliorer la qualité des politiques NordSud, mais aussi pour la mise à disposition des biens publics internationaux dont dépendent la sécurité et le bien-être du monde entier - et donc aussi des pays industrialisés.

\section{LES OBSTACLES À UNE COHÉRENCE DES POLITIQUES NORD-SUD}

La réponse à cette question dépend tout d'abord du caractère conscient ou non de l'incohérence. Dans le second cas, les obstacles sont à chercher dans le système de prise de décision et en particulier dans le sous-système de production et de circulation de l'information. Dans le cas d'un manque de cohérence voulu ou accepté, les obstacles sont plus difficiles à surmonter.

Les obstacles sont différents d'un pays industriel à l'autre en raison de la diversité de leurs intérêts et de la nature de leurs relations avec les pays en développement. Ils varient aussi en fonction du type de pays en développement concernés. Des conflits d'intérêts seront pas ou peu fréquents lorsqu'il s'agit d'un petit pays, «moins développé» et lointain, avec lequel les relations sont pratiquement limitées au champ de l'aide publique au développement. Par contre, les relations avec un pays en développement politiquement et économiquement important pour les pays industrialisés représentent des enjeux économiques et politiques de poids. Cette différence est particulièrement perceptible dans la

8. Un des exemples célèbres d'incohérence souvent repris dans la littérature est celui de l'exportation à des prix «cassés» de surplus de viande de bœuf de l'Union européenne vers des pays d'Afrique occidentale, exportation qui met en péril le développement de l'élevage dans ces pays soutenus par des pays de l'Union européenne (voir J. Forster and O. Stokke, op. cit., pp. 11, 139, 336)! 
politique de promotion des droits de l'homme où la flexibilité de la mise en œuvre est proportionnelle à l'importance politique et économique du pays concerné.

Identifier les obstacles requiert une analyse des causes de l'incohérences qui résident, dans la plupart des cas, dans des conflits d'intérêts. Dans une société démocratique, gérer ces conflits d'une façon responsable est une tâche cardinale du système politique; formuler et mettre en œuvre des politiques cohérentes est précisément ce qui confère sa crédibilité au pouvoir politique. Mais dans le domaine des relations Nord-Sud, les seuls conflits d'intérêts qui comptent vraiment sont ceux qui opposent les intérêts des pays en développement à ceux des pays industriels. On attend des gouvernements qu'ils défendent les intérêts nationaux; dans le cas d'un réel conflit entre les intérêts nationaux et ceux des pays en développement, il y a donc peu de doute qu'il sera tranché en faveur des premiers. On touche ici aux limites de la recherche d'une plus grande cohérence dans les relations Nord-Sud.

L'obstacle est de taille. On peut analyser son ampleur et sa nature selon deux axes, dont l'un mesure l'ampleur de l'intérêt à défendre (des intérêts spécifiques aux intérêts généraux), et l'autre l'horizon temporel (du court au long terme). Sur cette base, deux propositions - qui résument d'ailleurs la raison d'être de la coopération au développement - peuvent être formulées: les conflits d'intérêts sont nombreux dans le court terme et sur des questions touchant à des intérêts sectoriels; ils deviennent plus rares lorsqu'on se déplace vers le long terme et vers la prise en compte de l'intérêt national général.

Il est en effet largement reconnu aujourd'hui que le développement économique et social des pays à faible revenu constitue une condition indispensable à la gestion des problèmes globaux qui concernent l'ensemble de l'humanité. Indépendamment d'impératifs moraux, il est donc dans l'intérêt des pays industrialisés de voir le sort de la population de ces pays s'améliorer. Le PNUD a clairement présenté cette convergence d'intérêts dans son rapport de 1994 sur le développement humain: «Quel que soit l'endroit du monde où la sécurité des personnes est compromise, tous les autres pays du monde risquent d'en subir les effets. La famine, la maladie, la pollution, le trafic de drogue, le terrorisme, les conflits ethniques et la désagrégation du tissu social ne sont plus des phénomènes isolés, circonscrits à l'intérieur des frontières nationales: leurs conséquences touchent l'ensemble de la planète. $»^{9}$ Dans cette perspective, il est dans l'intérêt national des pays industriels de rechercher plus de cohérence dans les politiques Nord-Sud en vue de les rendre plus efficaces dans la promotion du développement.

Les exemples les plus fréquents de conflits d'intérêts spécifiques et à court terme proviennent souvent du commerce international. Ainsi il est généralement admis que l'aide liée n'est pas dans l'intérêt des pays en développement: elle complique singulièrement la gestion des programmes de développement, réduit la valeur réelle de l'aide fournie et peut concurrencer de façon déloyale les producteurs locaux ou régionaux; en outre, elle sert les intérêts particuliers de secteurs et de firmes peu compétitives dans le pays fournisseur d'aide. Le conflit d'intérêts est patent à court terme. A plus long terme cependant, on peut argu-

9. PNUD, Rapport mondial sur le développement humain 1994, Economica, Paris, 1994, p. 3. 
menter, à l'image de Catrinus Jepma ${ }^{10}$, que le liement de l'aide est contraire également à l'intérêt du pays fournisseur d'aide dans la mesure où le processus d'ajustement des firmes ainsi soutenues est retardé et qu'elles sont davantage occupées à maintenir le statu quo de leurs privilèges qu'à rechercher les moyens d'améliorer leur compétitivité.

Un autre exemple, plus important, est celui des politiques protectionnistes des pays industriels dans les domaines agricole et des textiles, qui s'opposent aux efforts des pays en développement pour développer leurs exportations. Même si l'Uruguay Round a marqué le début d'une politique de démantèlement de ces protectionnismes, le temps donné aux pays industriels pour procéder à leur ajustement structurel est bien plus long que celui dont disposent les pays en développement pour effectuer le leur. Les conflits d'intérêts sont dans ce cas d'une grande ampleur car ils touchent des pans entiers de l'économie et de la société. Ce n'est pas ici le lieu de discuter les mérites et les risques de la globalisation économique, mais on peut relever le manque de cohérence entre le discours et la pratique des pays industriels en matière de libéralisme économique.

Ces obstacles ne peuvent être surmontés et il existera toujours une part d'incohérence dans les politiques Nord-Sud. Cependant, il s'agit, par cette nouvelle approche, d'éviter autant que possible les incohérences non voulues ou acceptées et de disposer de mécanismes permettant de prendre les décisions en pleine connaissance des enjeux et des conflits d'intérêts.

\section{MÉCANISMES POUR PROMOUVOIR LA COHÉRENCE DES POLITIQUES}

Au sein d'un gouvernement, le ministère ou l'agence chargé de la coopération au développement a de toute évidence un rôle central à jouer dans la promotion de la cohérence des politiques. Il s'agit de situer sa place et son statut vis-à-vis des ministères clés tels que les Affaires étrangères, les Finances et l'Economie. Or, on constate un dilemme quant au degré d'autonomie dont devrait jouir l'administration de la coopération au développement par rapport à ces ministères clés. Un organisme relativement autonome peut sans doute poursuivre une politique avec une forte cohérence interne. Cependant, comme le montre l'exemple du Canada", une agence autonome aura certainement peu d'influence sur les autres politiques Nord-Sud. Inversement, une administration plus intégrée dans le processus décisionnel gouvernemental pourra participer à la formulation de toutes les politiques mais risquera d'être plus aisément instrumentalisée pour défendre des intérêts politiques ou commerciaux. Dans cette hypothèse, la situation est différente si l'administration de la coopération est elle-même un ministère de plein droit (exemple de l'Allemagne) ou intégrée dans un ministère clé (cas de la Suisse). L'étude de plusieurs cas montre qu'aucune formule ne semble recueillir un large consensus.

Une autre question, très pertinente dans le cas de la Suisse, est celle de savoir si la cause de la cohérence des politiques est mieux défendue par une concentra-

10. C. Jepma, «The Case for Aid Untying in OECD Countries», in O. Stokke (ed.), Foreign Aid towards the Year 2000: Experiences and Challenges, EADI Book Series 18, Frank Cass, London, 1996, pp. 246-247.

11. C. Pratt, «Greater Policy Coherence: A Mixed Blessing», in J. Forster and O. Stokke, op.cit. 
tion de toute la coopération au développement au sein d'une seule entité administrative ou par un partage des compétences entre plusieurs institutions. La formule de la concentration présente l'avantage de faciliter la circulation de l'information et la coordination des diverses activités. On peut cependant aussi argumenter qu'il est bon pour la promotion de la cohérence qu'une bonne connaissance des problèmes et des enjeux de la coopération au développement soit présente dans plusieurs ministères.

Aujourd'hui, par la diversité des problèmes de développement, pratiquement tous les ministères traitent de dossiers liés aux relations Nord-Sud. La coordination interministérielle devient complexe, ainsi que l'illustre l'organisation de délégations aux grandes conférences des Nations Unies (population, femmes, environnement, habitat, etc.). Les aspects Nord-Sud de ces problèmes représentent souvent les éléments les plus importants et les plus controversés de ces conférences et les ministères compétents pour ces dossiers n'ont pas toujours l'expertise nécessaire. Les mécanismes de coordination interministériels sont alors fortement mis à contribution.

Les relations Nord-Sud sont cependant une préoccupation constante d'un petit nombre de ministères: il s'agit des Affaires étrangères (le cas échéant de la Coopération), des Finances et de l'Economie. Ce sont donc les mécanismes de coordination entre ces ministères qui revêtent la plus grande importance; leur existence et leur efficacité représentent un bon indicateur de la volonté politique d'améliorer la cohérence des politiques Nord-Sud. Ces mécanismes doivent permettre une bonne circulation interministérielle de l'information sur tous les projets de décision entrant dans le cadre conceptuel de la cohérence des politiques. Ils doivent aussi donner au ministère «gardien de la cohérence » - en principe celui de la coopération au développement - la légitimité et la possibilité de se prononcer sur les effets d'un projet de décision sur le développement des pays concernés. Ils doivent enfin permettre aux autorités de prendre leurs décision en pleine connaissance de tous les éléments pertinents.

Cependant, la force et la constance de la volonté politique dépendent aussi de l'engagement d'autres acteurs dans le processus décisionnel politique. Les parlements ont un rôle central à jouer pour encourager le gouvernement à rechercher davantage de cohérence et pour suivre la mise en œuvre de cette politique $^{12}$. Les acteurs qui sont partie prenante ou qui disposent d'expérience dans ce domaine (ONG, secteur privé, milieux académiques) peuvent aussi jouer un rôle important. Leur participation en tant que conseillers dans le processus de prise de décision et dans le suivi des politiques peut contribuer à réduire les incohérences non voulues et amener le gouvernement à présenter ses décisions sous l'angle de leurs effets sur les pays en développement. Il s'avère donc aussi important de prendre en compte les mécanismes permettant d'une part au parlement, d'autre part à la société civile, de participer à la promotion de politiques plus cohérentes.

12. En Suisse, c'est une commission parlementaire qui, en 1990, demanda au Conseil fédéral d'établir des lignes directrices pour une politique de développement globale et cohérente. 
L'approche de la cohérence des politiques est certainement un pas important vers une plus grande prise en compte des intérêts des pays en développement - et de l'intérêt commun à long terme - dans les relations Nord-Sud. Cela est nécessaire car les relations entre pays industriels et pays en développement - en particulier les plus pauvres d'entre eux - continuent d'être fortement asymétriques.

Cette approche ne doit pas non plus susciter des attentes exagérées. Des conflits d'intérêts existent; ils continueront d'exister et de se régler selon les rapports de force. Il convient encore de souligner que l'engagement des pays membres du CAD pour cette approche est très variable. Jusqu'ici, ce sont surtout les pays scandinaves, les Pays-Bas, l'Allemagne et la Suisse qui se sont engagés sur cette voie. Encore faudra-t-il mesurer cet engagement sur le long terme et vérifier si la recherche de cohérence n'est pas qu'une mode passagère.

Selon les expériences réalisées dans plusieurs pays industriels ces dernières années, l'efficacité de cette approche dépend des conditions suivantes:

- existence d'une base légale claire définissant les objectifs de la politique de coopération au développement;

- existence de lignes directrices gouvernementales claires sur la cohérence des politiques, sur les objectifs des politiques Nord-Sud du pays, ainsi que sur les relations entre intérêts nationaux et promotion du développement;

- mise en place d'un mécanisme de coordination interministériel pour la formulation et le suivi de la mise en œuvre des politiques;

- participation des acteurs non gouvernementaux en tant que conseillers dans le processus de formulation et de suivi des politiques.

La cohérence des politiques devrait ainsi favoriser un processus de prise de décision plus systémique, qui

- mette en évidence l'impact des politiques sur les pays en développement et leur compatibilité, ou incompatibilité, avec les objectifs de la politique de coopération au développement ;

- rende plus transparent le processus de formulation et de mise en œuvre des politiques et par là encourage une analyse systématique et un débat public des conflits d'intérêts;

๖ promeuve une meilleure compréhension dans les milieux spécialisés, mais aussi auprès du grand public, des enjeux à long terme des relations Nord-Sud pour les pays industriels. 